\title{
Blockchain as Radical Innovation: A Framework for Engaging with Distributed Ledgers
}

\author{
Roman Beck \\ IT University of Copenhagen \\ beck@itu.dk
}

\author{
Christoph Müller-Bloch \\ IT University of Copenhagen \\ chmy@itu.dk
}

\begin{abstract}
Blockchain is an emerging technology that is perceived as groundbreaking. However, blockchain presents incumbent organizations with significant challenges. How should they respond to the advent of this innovative technology, and how can they build the capabilities that are necessary to successfully engage with blockchain? In this case study, we analyze how an incumbent bank deals with the radical innovation of blockchain. We find that blockchain as an innovation is unique, because its transaction cost-lowering nature requires cooperation not only on an intra-organizational, but also on an inter-organizational level to fully leverage the technology. We develop a framework illustrating how the process of discovering, incubating, and accelerating with blockchain can look like. Our research is one of the first case studies in the area; shedding light on the organizational challenges of incumbents as they engage with blockchain. The paper provides a blueprint for business executives in their endeavor of embracing blockchain technology.
\end{abstract}

\section{Introduction}

Blockchain technology receives a lot of attention in the information technology (IT) and financial industry these days, being referred to as being potentially the most promising technology in financial services ever [5,6,16,23]. Every major bank and financial institution is looking into the potential of applying this technology in different areas of their business, such as payment, stock trading, or similar transaction-based processes [2]. The expected gains are improvements in speed, security, and transparency along with a general reduction of transaction costs - just to name a few.

In addition, the long-term predictions suggest a remarkable, even revolutionary potential to redefine our entire financial system and to change some of the most fundamental structures of our economy and society [2,20,21]. To emphasize this, blockchain is compared by some to the invention of the Internet and its comprehensive impact on almost every industry $[5,6,14,16]$. Indeed, blockchain has made inroads into many industries since it first materialized in early 2009 as underlying technology of the cryptocurrency Bitcoin, with high expectations for its future. Blockchain can be defined in brief as a distributed ledger or list of data records of transactions that may involve any kind of value, money, goods, property, or votes. The blockchain is shared in a decentralized network of computers and based on mathematics and advanced cryptography, where each transaction can be verified by the entire network that can be either public or private. In this way, blockchain technology challenges any business model that relies on third parties for trust and verification such as insurance companies or banks. Evidently, this puts a remarkable pressure on incumbent organizations, whose operations are built upon trust-securing models such as in the case of banks which act as trusted third-parties and those have a central role in the economy as we know it.

Clearly, with blockchain and distributed ledgers, the rules of the game are changing, adding pressure on financial institutions to be more innovative to reinvent their existing business models. Incumbent banks have to rethink what their value propositions in the future might be.

In the literature on innovation management, this phenomenon of ascribing a very large promise to a technological opportunity is often referred to as "radical innovation" and is a widely addressed and a persistent theme in innovation studies [1,3,8,9,18,25]. In particular, radical innovation is often perceived to be a managerial challenge in established organizations, because it involves high uncertainty and unpredictability. Thus, established organizations have to build the necessary capabilities to manage, absorb, and adapt new technologies such as blockchain [19]. In this process, established organizations increasingly team up with external parties to stay in touch with the latest trends and 
avoid falling behind in the innovation race [26]. This way of working calls for these actors to lower their organizational boundaries to allow knowledge to freely flow within and between organizations.

In this paper, we explore the impact blockchain has as radical innovation on incumbent industries. The financial services industry is taking blockchain very seriously now and is developing know-how and capabilities in that area. However, it is unclear how banks and incumbent organizations in general will deal with such a radical technological innovation that has the potential to disrupt large parts of their traditional business models. How do incumbent organizations respond to blockchain as radical innovation? How can they build the needed competencies to rethink their current business models in the light of radical innovation?

In order to answer our research questions, we conducted a case study within a large international bank that is engaged with blockchain along the lines of discovery, incubation, as well as acceleration. We will illuminate how they can build the competences that are necessary to rethink existing business models in the light of blockchain.

The paper is structured as follows. Section 2 explores the foundational literature on both blockchain technology and radical innovation. Section 3 describes the case as well as the methodology. Section 4 presents the findings of the case study. Section 5 discusses the insights gained and concludes.

\section{Literature Background: Blockchain as Radical Innovation}

\subsection{Blockchain and its Components}

Blockchain is regarded as a technology that has (and will have even more in the future) a radical impact on the financial services sector. In the following, we will briefly discuss what blockchain actually is: Blockchain was first introduced in the source code of Bitcoin. It is argued that Bitcoin used blockchain of the first generation, called blockchain 1.0, which was only designed to support crypto currencies. The blockchain of the second generation moved away from Bitcoin and its single focus on cryptocurrency and allows all kinds of transactions to be coded into a freely programmable blockchain, such as the Ethereum blockchain, where one can implement business logics in so-called smart contracts [2]. Thus, blockchain 2.0 has an extended functionality compared to its predecessor, making it a generically programmable platform that can serve as infrastructure for all kinds of blockchain secured applications [20]. In other words, blockchain 2.0 offers a digital, open source peer-to-peer transaction system where ledgers, or more precisely databases, are decentralized and distributed across a network of users $[15,20]$. The database consists of chains of blocks, each containing a list of transactions. To validate the transactions within the block, the block operates as advanced cryptographic puzzle that has to be solved. This approach is called proof of work and relies on so-called miners to solve these puzzles, for instance, for the Bitcoin blockchain [20,28]. When the puzzle for a block of transactions is solved, a new block for new transactions is generated and added to the chain. Each block is placed in a chronological order on the blockchain; thus, the blockchain contains the complete history of all transactions.

The highly cryptographic design of the blockchain technology makes it practically impossible to reverse or tamper with transactions $[15,20,22]$. Moreover, all participants within the system have a personal key or signature that is used when creating a transaction. This key makes it possible to account for which user created a given transaction and to whom that specific transaction was sent to $[15,20]$. Also, the combination of the ledger being distributed and validated across the network makes it possible to assign any asset to any user, and that a single asset cannot be sent more than one time. In other words, this prevents double-spending assets which in effect makes it possible to track ownership for a certain asset at any point.

Furthermore, because blockchain technology is a peer-to-peer technology, it operates on a network of users, who are also called nodes. The technology is reliant on the network of nodes to work together to validate transactions $[15,20]$. In principle, all who engage in the blockchain can see all transactions happening on the blockchain as well as review past transactions. The blockchain technology can be utilized in two different ways; public blockchains and private blockchains, also called unpermissioned and permissioned [4,13]. In public or unpermissioned blockchains, everyone who wishes to engage in the network can openly see all transactions. The technology is transparent and all who wish to engage in making transactions on the blockchain can do so. In contrast, private or permissioned blockchains are closed and only accessible for a selected few who have permission to engage in the blockchain. The transparency is therefore only given for permissioned participants, which is making it challenging to handle data that requires a certain privacy, as it is the case with, for instance, customer data in the finance sector. Like traditional legal documents, smart 
contracts in unpermissioned or permissioned blockchains comprise rules, rights, and consequences. However, unlike a traditional contract, a smart contract can be supplied with information, which can automatically be processed through the predefined rules, and take action upon in regards to the defined consequences [2]. Moreover, as smart contracts operate on a blockchain, they submit to all the specifications of the blockchain technology. This means that not only does the contract operate automatically, it is also distributed across the network and operates on the premise of the aforementioned structure of the blockchain technology.

The advent of blockchain has been compared with the invention of the Internet, having a huge potential for creating groundbreaking transformations within a number of industries [16,23]. In innovation management literature, such a phenomenon of groundbreaking change is often referred to as radical innovation [18], that will be discussed next.

\subsection{Blockchain as Radical Innovation}

Radical innovations are discontinuous events that are often direct results of research and development [9], having a disruptive impact on existing business models [3,9]. Radical innovations often represent revolutionary changes in technology [8]. In other words, a radical innovation outdates existing technologies and practices within a given field [25]. What distinctively differentiates radical innovation from incremental innovation is that incremental innovations are typically adding new functionalities to existing technologies, while radical innovations are new technologies with new functionalities [3]. Radical innovations are not only promising potential rewards for organizations, but are also risky since such fundamental technological changes require to adapt old or develop new organizational competencies to perform differently $[1,8]$. It can be assumed that the radical innovation of blockchain will trigger significant organizational changes through the introduction of new business models and organizational practices. As such, radical technological innovation are particularly difficult for established organizations to manage $[7,11,12,19]$. Radical innovations come with methods and materials that are new to the incumbent organization, requiring knowledge that typically has to be absorbed from external sources and combined with established organizational knowledge [11].

Radical innovation involves a high level of market, technical, resource, and organizational uncertainty, which can potentially be converted into long project maturity durations and unpredictable development. Therefore, organizations need three sets of competencies to manage the particular fields of radical innovation [19], namely discover, incubate, and accelerate capabilities. As organizations decide to develop radical technical innovations, they are bound to stretch the boundaries of what they already know, and in doing so, accessing market partners and expertise in different environments enables the company in developing their capabilities for radical innovation.

The first set of competencies is discovery. This refers to capabilities involving activities that create, recognize, elaborate, and articulate radical innovation opportunities [19]. In relation to the activities, certain skills are required to carry these out. These skills are exploratory and conceptualization skills, such as conducting basic research and internal and external hunting for opportunities. Because the discovery competencies include both invention and discovery of radical innovations, it means that discovery involves creating or discovering something different and previously unknown to the inventors. In other words, discovery is about being or becoming aware of innovations that were previously unknown to the company. Therefore, a mature set of discovery competencies not only involves internal research and development, but also activities focusing on acquiring external knowledge [19].

The incubation competencies include activities for maturing radical opportunities into business proposals. Whereas discovery competencies create or recognize opportunities, incubation competencies create hypotheses of what a given opportunity could become within the market [19]. These hypotheses include a potential business model, a hypothesis of what the market could look like and what the technological platform could enable in the market. Furthermore, incubation is for testing out these hypotheses in the market with prototypes. The skills needed are experimentation and interaction skills.

The acceleration competencies involve activities for developing the business proposal to stand on its own in relation to other business platforms in the ultimate receiving unit [19]. This means focusing on building the proposed business to a level of predictability so that sales and operations can be somewhat calculated. The main skill needed for this is exploitation, including activities such as investment in building up the business and its underlying infrastructure while keeping focus on responding to market demands. Furthermore, focus is also upon creating processes for manufacturing, customer contact, support, and more. In other words, the focus here is to establish the full functioning business, and once the radical innovation generates 
returns, it will be submitted to existing businesses or create ground for its own business unit [19].

\section{Case Description and Methodology}

Our case company is one of the leading investment banks in the world, with more than 100,000 employees. In early 2014, some of its senior executives became aware of blockchain technology. While they agreed early on that the impact of blockchain on the banking sector might be significant, they needed to promote the technology within the firm and to build the capabilities to engage with blockchain. In addition, they needed to assess if the bank would be able to capture value from using blockchain technology. The bank managers in charge of the blockchain initiative were well aware of the criticality and the uncertainty that the advent of blockchain technology poses for their business, and thus developed a rich understanding of the technology and its business implications. Hence, the case company offers a unique opportunity to elicit interesting insights to deepen our understanding how incumbents cope with blockchain as a radical innovation. Moreover, the same circle of senior executives was driving the blockchain initiative within the firm over the whole period and were thus able to paint a comprehensive picture of the entire array of blockchain-related activities since 2014, allowing us to gain a historical overview of the whole process of managing the radical innovation that blockchain represents.

We conducted a single-case study to analyze how incumbent organizations in the financial sector manage radical innovation such as blockchain within their organizations. Given the scarcity of research on the blockchain and management of radical innovation in large organizations, our goal was to build a process model, using grounded theory techniques in case study research [24]. The case research we conducted took place within one of the largest investment banks in the world, making the organization particularly suitable for investigating blockchain and the phenomenon of interest, namely how to manage radical technological innovation.

Our primary data collection consisted of 5 interviews conducted in April 2016 at two different international sites of the investment bank. The interviews were conducted in English, digitally recorded, and subsequently transcribed and complemented by the extensive notes we took during each interview session [29]. Each interview lasted on average 1 hour, although we also had a 'long interview' with the head of the blockchain development unit, which lasted longer than 2.5 hours (see Table 1). Interviews were conducted in an openended and semi-structured manner. Our data sampling was closely aligned with our pre-conceived understanding about radical innovation, but otherwise open to allow for the analysis and emergence of new theoretical insights [24]. Our primary data collection was complemented by observations and informal face-to-face discussions during our field research at the bank's venues.

Table 1. Interviews for case study

\begin{tabular}{|l|l|}
\hline Interviewee & $\begin{array}{l}\text { Duration of } \\
\text { Interview }\end{array}$ \\
\hline Head of Technology Strategy & $01: 02$ \\
\hline Head of Innovation Lab & $01: 05$ \\
\hline Head of Debt Capital Markets & $01: 13$ \\
\hline Head of Blockchain & $01: 05$ \\
\hline Head of Digitization & $02: 40$ \\
\hline
\end{tabular}

In total, more than 90 pages of transcriptions and notes were created during the analysis process in May and June of 2016. In addition, for triangulation purposes, secondary data was collected and analyzed, such as presentation slides and a brief blockchain use case description. This information helped us to construct the evolution of events and activities in the blockchain development unit in 2014 and 2015, as well as the decision logic and development of blockchain-related competencies. Our methodology for investigating the case was 'pluralist,' meaning that we engaged in different research activities [17]. Our approach was also consistent with Mingers (2004) who advocates for a pragmatic approach in which researchers embrace different research perspectives with the aim of generating "a useful model of reality" [27]. We also followed the principle of 'emergence' from grounded theory: grounded theory must 'fit' the data under study, and it must meaningfully explain the behavior under study [10]. In addition, techniques were selected and used that helped us increase the theory's scope and the degree of conceptualization, for instance, by treating literature about radical innovation as additional data points that we used for the data analysis [24].

Our research process started with formulating the problem [27], designing the case study [30], and engaging in intertwined data collection and analysis to theories from our data. However, we did not follow all grounded theory method recommendations, for example, we did not apply different coding approaches, in part because we only conducted five interviews with a rather narrow scope on radical innovation management in the case of blockchain. 


\section{Empirical Data and Analysis}

\subsection{Discovery}

The discovery process is initiated by the recognition of the radical innovation. The bank's interest in blockchain was triggered by the rise of Bitcoin, which the firm's senior executives sensed required further scrutiny:

And the reason I got interested in it [blockchain], was I was in charge of product management for payments, and I go back to the beginning of 2014, and [...] people were talking about Bitcoin all of the time, and there was a curiosity about whether Bitcoin is going to be the currency of the future. And so, somebody had to take a look at it from our management team, and since I was [...] the person most associated with the technical part of it, I got that job basically. So I looked into [...] Bitcoin [...], and basically came to the conclusion that it was not very interesting as a currency, but actually [it was the] underlying technology [that was] quite interesting. " [Head of Blockchain]

Following an external regulatory event, the same executive became aware of the potential concrete applicability of blockchain technology for the financial sector shortly thereafter. This illustrates the importance of not only being aware of a radical innovation, but also of sensing its relevance for the own business:

A few months later I became much more interested in it [blockchain] because of something that happened from a regulatory perspective. And I was thinking, [...] how do you figure out who the client of a client is? And at that point I realized [...]. Blockchain [...] could actually work for that, because then I would have a ledger with [...] complete transparency. [Head of Blockchain]

Later in 2014, the future Head of Blockchain met a fellow executive interested in blockchain. They jointly decided to further pursue blockchain. To stimulate interest within the bank, they reached out both to senior executives of the firm, as well as to its other employees:

And so we then immediately had this connection, and I wrote a white paper in May of 2014 to try and get [bank name] management, particularly on the investment banking side, educated on what these things meant. By the end of that summer [the other executive and] I cohosted a distributed ledger internal working group mainly from people from global markets, from the innovation lab, and from the transaction bank, and that became our internal community of enthusiasts, and it was enthusiasts, because it was nobody's job at this point, and it went from there. [Head of Debt Capital Markets]

Building a community of volunteers allowed engaging with blockchain without having to acquire financial resources. Employees from different business units got together in their spare time. They were only involved because they were intrinsically motivated by the complexity and novelty of blockchain technology:

The most important thing that we've used as an enabler to try and cover that has been identifying the people in the organization who-and I wish I could put this more elegantly, but I promise you it's truewho will work on this in their spare time. They will do this, because it's intellectually interesting, because they like to know what's happening on the cutting edge, and it is genuinely surprising the level of commitments that people will demonstrate and how passionately they feel about it as a topic when they are allowed to innovate in this way. [Head of Debt Capital Markets]

\subsection{Transition from Discovery to Incubation}

Evolving the opportunity the blockchain posed for the bank further into a business proposition was contingent upon acquiring funding to allow for experimentation. The firm could hence move on to the incubation phase:

So we basically got the COO to kind of write us a very small check to perform a small experiment inside the Innovation Labs, and we then had the problem of who is going to work in it, because all of us had fulltime jobs. [Head of Blockchain]

After securing the funding, the executives decided to bring in the right experts to embark on experimentation. Bringing in the future users of the radical innovation was thought be helpful to manage resistance later on, in the transition from incubation to acceleration:

As far as adoption by the organization, the earlier you bring in more of the business, the easier the journey in, because you're not having to now sell your more formed solution to other parts of the bank 
and they don't feel as connected with it as you may feel [Head of Technology Strategy]

Even though the incubation phase would rely on volunteers, akin to the discovery phase, the funding also allowed for acquiring additional external capabilities. The bank decided to collaborate with startups, not with established vendors, to achieve significant cost savings:

But so, we looked for external vendors to support. We have a lot of knowledge in-house. We had a lot of business and technology knowledge, and architecture knowledge. What we didn't necessarily have anyone who could, you know, code the theory in. [...] we had all of the usual suspects on a big scale come in and then we got some startups. And it was intriguing, the stark difference between the money that the startups were looking for, which is effectively cover costs. The systematically larger company it was make a, you know, 60\% margin. So we opted to go with the startups. [Head of Innovation Lab]

After acquiring the funding, as well as bringing both internal and external expertise together, having the right environment for innovation in place was the last step before incubation began:

So we got the funding, got a whole bunch of different experts together. The [innovation lab] had the space to do it [to conduct experimentation], and they had just been set up. So they had a whole process, which helped us identify the right people to work with and how we should work with them. [Head of Blockchain]

\subsection{Incubation}

To allow for experimentation, the blockchain team designed use cases. Executives argued that the use cases allowed for running simulations and to assess how a blockchain-based solution would perform compared to a legacy solution. In addition, they tried to avoid overtly complex use cases, to focus on blockchain itself:

What we needed was a simulation to be able to test our existing environment against a prospective environment using a new technology, and we looked to use cases where the complexity and the level of digitization in the existing environment was reasonably low, transaction volumes were reasonably low, and that we didn't need to worry about externalities, and by that I mean clearinghouses, exchanges, trade reporting, and so forth. [Head of Debt Capital Markets]

The goal of mitigating potential resistance was likewise behind the decision the select corporate bonds as a use case:

The other element was we needed to pick a use case [...] our internal stakeholders [...] considered to be nonthreatening, right? That's actually quite important when we're trying to do something disruptive, right? [Head of Debt Capital Markets]

After the use case had been selected, the bank implemented a blockchain-based representation of corporate bonds:

Then we started to document the trade work flows that made up the life cycle of those products and then see how close we could get to replicating them, using distributed ledgers. [Head of Debt Capital Markets]

Going forward, the managers assessed the viability of the use case using hypothesis-driven experimentation:

And then, we executed the proof of concept with a very clear hypothesis [...] [we assessed] business outcomes and benefits within three months, which, again, is quite a record. [Head of Innovation Lab]

\subsection{Transition from Incubation to Acceleration}

The bank was still in the incubation phase, as the following quote illustrates, and was not moving toward acceleration yet:

[...] We're not yet at a point where it is [...] a commercial model. [Head of Debt Capital Markets]

However, executives already had a vision on how to make the shift from the incubation to the acceleration phase. They argue that the radical innovation will only be adopted if resistance can be overcome and if the adoption proves valuable for the firm:

And so our, the lab engagement gets smaller and smaller and smaller, as we go through. And the business [...] [becomes more] involved in this to a point where they are doing the final adoption. They're deploying it. They're managing the environment. The technology is alive. It's providing 
the value to the organization. [...] So this bit, the adoption, is the hardest part of it. [...] You can do lots of experimentation, but [the radical innovation will not be adopted] unless there's a part of the organization that wants to take this in, and unless it shows real value. [Head of Technology Strategy]

\subsection{Acceleration}

As argued above, the bank had not entered the acceleration phase yet. The bank was still at the incubation stage, conducting experiments and trying to foster organizational learning. The commercialization of blockchain and thus acceleration was expected to happen in the next few years.

\subsection{Fading Boundaries and Decentralization}

From an overall perspective, the efforts to engage with blockchain as a radical innovation led to fading boundaries both intra- and inter-organizational. Blockchain triggered cross-functional collaboration between different business units, such as technology, legal, and sales units. In addition, vendors were also heavily involved in the process. Hence, boundaries between organizations were also starting to disappear:

So [there are] fading business boundaries. You are heavily [involved] with your partners and with your vendors, it's not exactly clear anymore where you company ends, and where the [...] vendor company starts. " [Head of Blockchain]

Moreover, this trend toward fading boundaries is even welcomed by the employees:

Now people [from different business units] are coming together on a frequent basis, and nobody needs to be forced to go to any of those meetings. Everyone goes, because they want to go. [Head of Debt Capital Markets]

In addition, the blockchain initiative was also characterized by a high degree of decentralization:

Everybody is sort of informed, [...] has an opinion. And then this [can be seen] in the light of kind of knowledge work, as [...] everybody is sort of an expert in his, or her own field, makes it more and more complicated to respond to run the company, and to respond agile to [this] kind of issues. Now we have technologies, which are kind of accelerating this trend, like social media, or Blockchain, where the decentralization is kind of given more emphasis, rather than the centralization right. [Head of Blockchain]

\section{Discussion}

Our findings illustrate that firms engage with blockchain along the lines of discovery, incubation, and acceleration. While blockchain is a rather new phenomenon, our case shows that there are firms that in their blockchain initiatives already moved beyond discovery, are fully immersed in incubation, and are beginning to consider acceleration. In line with the theory we also find that the transitions between these three phases need to be considered to successfully engage with blockchain. Both the transition from discovery to incubation and from incubation to acceleration are key to initiate the respective next innovation phase. As our case illustrates, there are several key activities that are key to comprehensively engage with blockchain from discovery to acceleration (see Figure 1).

The discovery phase is initiated by the recognition of blockchain. However, recognizing the existence of blockchain is not sufficient. It is also necessary to realize that blockchain might be of relevance for the business. Subsequently, this finding needs to be communicated to stimulate interest within the firm. This may result in a community that is intrinsically motivated to engage with blockchain. The members of this community can foster organizational learning by educating themselves.

The new insights gained in this process can be used to initiate the transition from discovery to incubation, which is marked by the acquisition of financial resources. The incubation phase is reliant upon funding, since experimentation is usually associated by significant financial cost. There are also organizational enablers for the incubation phase: an innovation laboratory can offer the right environment for experimentation. It can also serve as an environment for collaboration of different business units as well as external vendors, which is crucial since diverse knowledge needs to be merged to successfully engage with blockchain. Bringing in the owners of business process early in the process can also be a tool to mitigate early on potential resistance to blockchain. 


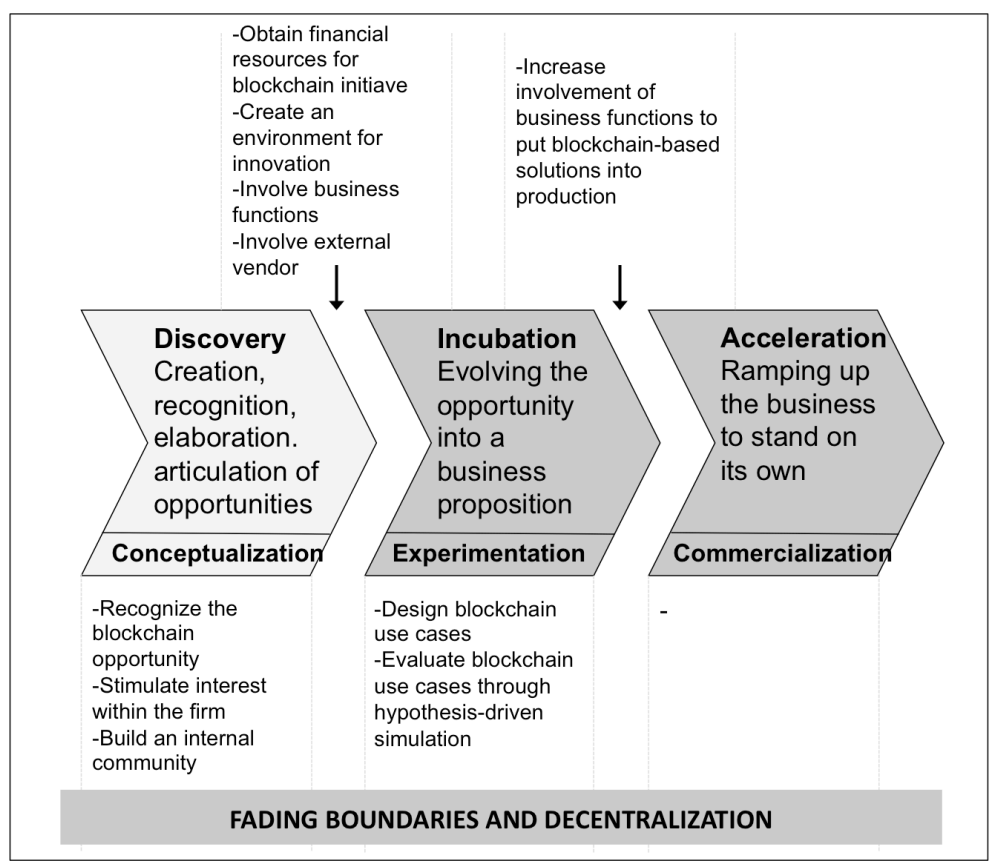

Figure 1. Blockchain Innovation Process (derived from [19])

The incubation phase is contingent upon having use cases that can be subject to experimentation. The use cases should allow for running simulations so that the effectiveness of blockchain-based solutions can be compared to legacy solutions. Moreover, use cases should be simple enough to enable rapid prototyping iterations, as well as focusing the impact of blockchain technology itself. They should also be chosen in a fashion that minimizes potential resistance. After the use cases have been designed, they can be implemented as a blockchain-based solution. Subsequently, the solutions can be subject to hypothesis-driven experimentation and can be assessed against predefined performance indicators.

To successfully facilitate the transition from incubation to acceleration, the involvement of the innovation laboratory needs to be reduced, while the business process owners need to take over the blockchain-based solution. However, a blockchainbased solution will only be adopted if resistance can be overcome and if it proves valuable for the firm.

Most firms engaging with blockchain have not entered the acceleration phase yet. However, the success of the commercialization of blockchain-based solutions is also contingent on the discovery and incubation phases, because these phases play an important role in blockchain-related organizational learning. Moreover, potential resistance to blockchain can be mitigated early on.
Blockchain initiatives are characterized by fading boundaries between the different actors that are involved. Both on an intra-organizational as well as inter-organizational level, blockchain initiatives require close collaboration since the necessary competences are scarce and widely dispersed. Moreover, inter-organizational cooperation is key to fully leverage the transaction-cost lowering potential of blockchain. Blockchain initiatives also exhibit a high degree of decentralization, because they require collaboration among a wide array of experts, from areas such as information technology and law.

\section{Implications and Limitations}

Our paper has implications for both practitioners and researchers. For practitioners, we illustrate how banks can engage with blockchain in a timely and sustainable fashion. We illuminate how they can build the competences that are necessary to rethink existing business models in the light of blockchain, by embracing fading boundaries between different business units as well as different organizations. For researchers, we analyze how incumbents can pursue blockchain as a radical innovation, by addressing the three phases of discovery, incubation, and acceleration.

A limitation of our research is the rather limited number of interviews we conducted. Although we had access to key decision makers and archival data, 
more interviews might have helped us to get a more nuanced view. In addition, this case study has been conducted at a very early stage of blockchain developments within organizations. As such, we can only shed light on a very important development at a very early stage of how incumbent organizations deal with blockchain as radical innovation. Future research should both focus on the induced organizational change due to blockchain as well as take a more differentiated view, such as the impact of blockchain as new transaction infrastructure versus the impact of blockchain as driver for new products and services.

This paper is the first to illustrate how incumbents can engage with the radical innovation of blockchain. By conducting a case study with a leading global bank, we elicit insights into the process of engaging with blockchain. We find that blockchain requires lowering boundaries both within and across organizations. Incumbents can address blockchain technology by engaging in discovery, incubation, and acceleration, and by carefully managing the transitions between these three stages.

\section{References}

[1] Adams, R., Bessant, J., and Phelps, R. Innovation management measurement: A review. International Journal of Management Reviews 8, 1 (2006), pp. 21-47.

[2] Beck, R., Czepluch, J.S., Lollike, N., and Malone, S.O. Blockchain - The Gateway to Trust-free Cryptographic Transactions. ECIS 2016 Proceedings, (2016), pp. 1-14.

[3] Betz, F. Innovation and Economy. In Managing Technological Innovation: Competitive Advantage from Change. John Wiley \& Sons, Inc., Hoboken, New Jersey, 2011.

[4] Bhaskar, N.D. and Chuen, D.L.K. Bitcoin Mining Technology. In Elsevier Inc., 2015.

[5] Buehler, K., Lemerle, M., Chiarella, D., Lal, A., Heidegger, H., and Moon, J. Beyond the Hype: Blockchains in Capital Markets. 2015.

[6] Courtneidge, R. and Buelli, F. Blockchain and Financial Services - Industry snapshot and possible future developments. 2015.

[7] Danneels, E. Disruptive Technology Reconsidered: A Critique and Research Agenda. Journal of Product Innovation Management 21, (2004), pp. 246-258.

[8] Dewar, R. and Dutton, J. The Adoption of Radical and Incremental Innovations: An Empirical Analysis. Management Science 32, 11 (1986).
[9] Freeman, C. and Perez, C. Structural crises of adjustment, business cycles and investment behaviour. In G. Dosi, C. Freeman, R. Nelson, G. Silverbeg and L. Soete, eds., Technical Change and Economic Theory. Pinter, London, 1988.

[10] Glaser, B.G. and Strauss, A.L. The Discovery of Grounded Theory: Strategies for Qualitative Research. AldineTransaction, New Brunswick, London, 2008.

[11] Hill, C.W.. and Rothaermel, F.T. The Performance of Incumbent Firms in the Face of Radical Technological Innovation. The Academy of Management Review 28, 2 (2003), pp. 257-274.

[12] Kline, S. and Rosenberg, N. An Overview of Innovation. In R. Landau and N. Rosenberg, eds., The Positive Sum Strategy: Harnessing Technology for Economic Growth. National Academy Press, Washington, 1986, pp. 275-305.

[13] Kosba, A., Miller, A., Shi, E., Wen, Z., and Papamanthou, C. Hawk: The Blockchain Model of Cryptography and Privacy-Preserving Smart Contracts. 2015.

[14] Lepak, D.P., Smith, K.G., and Taylor, M.S. Value creation and value capture: A multilevel perspective. Academy of Management Review 32, 1 (2007), pp. 180194.

[15] Mas, I. and Chuen, D.L.K. Bitcoin-Like Protocols and Innovations. In Handbook of Digital Currency. Elsevier, 2015.

[16] McLean, J. Banking on Blockchain: Charting the Progress of Distributed Ledger Technology in Financial Services. 2016.

[17] Mingers, J. Combining IS Research Methods: Towards a Pluralist Methodology. Information Systems Research 12, 3 (2001), pp. 240-259.

[18] O'Connor, G. and McDermott, C. The human side of radical innovation. Journal of Engineering and Technology 21, (2004), pp. 11-30.

[19] O'Connor, G.C. Open, Radical Innovation: Toward an Integrated Model in Large Established Firms. In H. Chesbrough, W. Vanhaverbeke and J. West, eds., Open Innovation. Researching a New Paradigm. Oxford University Press, 2005, pp. 62-81.

[20] Swan, M. Blockchain: Blueprint for a new economy. O’Reilly Media Inc, Sebastopol, CA, 2015.

[21] Tapscott, D. and Tapscott, A. Blockchain Revolution: How the Technology Behind Bitcoin Is Changing Money, Business, and the World. Penguin Random House, New York, 2016.

[22] Tarasiewicz, M. and Newman, A. Cryptocurrencies as 
Distributed Community Experiments. In Handbook of Digital Currency. Elsevier Inc., 2015.

[23] Taylor, S. Blockchain: understanding the potential. 2015.

[24] Urquhart, C., Lehmann, H., and Myers, M. Putting the 'Theory' Back into Grounded Theory: Guidelines for Grounded Theory Studies in Information Systems. Information Systems Journal 20, 4 (2010), pp. 357-381.

[25] Utterback, J. and Acee, H. Disruptive Technologies: An Expanded View. International Journal of Innovation 9 , 1 (2005), pp. 1-17.

[26] Vanhaverbeke, W. The Interorganizational Context of Open Innovation. In H. Chesbrough, W. Vanhaverbeke and J. West, eds., Open Innovation. Researching a New

Paradigm. Oxford University Press, 2005, pp. 205-219.

[27] Van de Ven, A.H. Engaged Scholarship: A Guide for Organizational and Social Research. Oxford University Press Inc, New York, 2007.

[28] Vigna, P. and Casey, M.J. The Age of Cryptocurrency. How Bitcoin and the Blockchain Are Challenging the Global Economic Order. Picador, St. Martin's Press, New York, 2015.

[29] Walsham, G. and Sahay, S. Gis for District-Level Administration in India: Problems and Opportunities. MIS Quarterly 23, 1 (1999), pp. 39-65.

[30] Yin, R.K. Case Study Research. SAGE Publications, Thousand Oaks et al., 2014. 